\title{
Reduced expression of MTUS1 mRNA is correlated with poor prognosis in bladder cancer
}

\author{
JUN XIAO $^{1 *}$, JUN-XING CHEN $^{2 *}$, YU-PING ZHU ${ }^{1}$, LIN-YU ZHOU ${ }^{1}$, QI-AN SHU ${ }^{1}$ and LING-WU CHEN ${ }^{2}$ \\ ${ }^{1}$ Department of Urology, Anhui Provincial Hospital, Anhui Medical University, Hefei, Anhui; \\ ${ }^{2}$ Department of Urology, The First Affiliated Hospital, Sun Yat-sen University, Guangzhou, Guangdong, P.R. China
}

Received October 21, 2011; Accepted March 27, 2012

DOI: $10.3892 / \mathrm{ol} .2012 .673$

\begin{abstract}
Mitochondrial tumor suppressor 1 (MTUS1) is a newly identified candidate tumor suppressor gene. Previous studies have demonstrated that the expression status of MTUS1 is altered in several types of tumors. However, its clinical significance for bladder cancer patients remains undetermined. In this study, we detected the expression of MTUS1 mRNA in bladder tumors and paired normal samples obtained from 5 patients using semi-quantitative reverse transcription-polymerase chain reaction (RT-PCR). A significant downregulation of MTUS1 mRNA expression was observed in the tumor tissues compared with the corresponding normal bladder tissue $(\mathrm{P}<0.001)$. We further tested the expression of MTUS1 mRNA in 55 bladder cancer tissues and 10 adjacent normal bladder tissues by quantitative real-time RT-PCR. Correlations between MTUS1 and clinicopathological features and prognosis were investigated by statistical analyses. The results showed that MTUS1 expression was correlated with tumor grade, stage, size and number $(\mathrm{P}<0.001, \mathrm{P}<0.001$, $\mathrm{P}=0.034$ and $\mathrm{P}=0.029$, respectively). Patients with low levels of MTUS1 mRNA expression had a poor prognosis compared with those with a high expression $(\mathrm{P}<0.001)$. Univariate and multivariate logistic regression prognostic analyses revealed that MTUS1 mRNA was an independent prognostic factor for disease-free survival in bladder cancer $(\mathrm{P}<0.05)$. In conclusion, these data suggest that MTUS1 is significant in the progression of bladder cancer and that the status of MTUS1 mRNA expression is a novel prognostic marker for predicting bladder tumor disease-free survival.
\end{abstract}

Correspondence to: Dr Ling-Wu Chen, Department of Urology, The First Affiliated Hospital, Sun Yat-sen University, No. 58, ZhongShan 2nd Road, Guangzhou, Guangdong 510080, P.R. China E-mail: xjdoctor@126.com

*Contributed equally

Key words: mitochondrial tumor suppressor 1, prognosis, survival, bladder cancer

\section{Introduction}

Bladder cancer is a common disease, with an estimated 386,300 new cases and 150,200 mortalities occurring in 2008 worldwide (1). The disease ranks ninth in worldwide cancer incidence and is the seventh most common malignancy in men (2). In China, bladder cancer is the most common malignant neoplasm of the male urogenital system and the incidence of bladder cancer has shown an upward trend in the past two decades (3).

Tumorigenesis is a multistep process during which cells acquire genetic alterations that drive the progressive transformation of normal cells into malignant cells. Genetic events which result in the progression of bladder cancer are complicated and poorly understood. Despite being a common cancer worldwide, the management of bladder cancer currently relies primarily on clinical staging and histopathological parameters. Of newly diagnosed bladder cancer cases, $70-80 \%$ present with non-muscle-invasive disease and, despite endoscopic and intravesical treatments, $50-70 \%$ recur and $10-30 \%$ progress to muscle-invasive disease (4). Thus, it is of great value to explore the mechanism of bladder cancer genesis and progression and to identify the molecular markers that predict bladder cancer recurrence and progression.

Mitochondrial tumor suppressor 1 (MTUS1), also known as ATIP [angiotensin II receptor subtypes 2 (AT2)-interacting protein], is a newly identified candidate tumor suppressor gene (5). Multiple splice transcript variants which encode different isoforms have been identified in this gene. Previous findings have demonstrated that MTUS1 was reduced or lost in a number of tumors, including pancreatic and ovarian tumors $(5,6)$. MTUS1 maps to chromosome 8p21.3-22, a region frequently deleted and associated with the progression of disease in breast, colorectal, lung, ovarian, renal, prostate and bladder cancer (7-13).

As yet, no study has investigated the expression of MTUS1 in bladder cancer. Thus, we explored the expression of MTUS1 and its clinicopathological and prognostic significance in human bladder cancer in this study.

\section{Materials and methods}

Study population and sample collection. There were 55 patients in the study, all of whom had bladder transitional cell carcinoma (BTCC) and were treated at our two institutions 
between January 2009 and March 2011. The patients included 44 males and 11 females aged between 27 and 81 years (mean, 60.5). The patients were histopathologically diagnosed as having BTCC, newly diagnosed and untreated, and had no history of any other tumor. We excluded carcinoma in situ from our study. Sixteen patients underwent radical cystectomy, 4 patients underwent partial cystectomy and 35 patients underwent transurethral resection of the bladder tumor (TURBT). This study was approved by the medical ethics committee of our hospitals and written informed consent was obtained from all patients. The surgically removed tumors were immediately frozen in liquid nitrogen and maintained at $-80^{\circ} \mathrm{C}$ until RNA was extracted.

A total of 10 normal bladder tissue samples $(5 \mathrm{~cm}$ distance from the tumor) were surgically excised from different patients who underwent radical cystectomy and were also stored at $-80^{\circ} \mathrm{C}$. The patients who underwent partial cystectomy or TURBT received intravesical mitomycin C (MMC) or pirarubicin (THP) instillations following surgery once weekly for the first 8 weeks and then monthly up to 1.5 years. Cystoscopy was performed at 3 -month intervals during the first 2 years and 6 -month intervals after 2 years. The mean follow-up period for the 55 patients was 14 months (range, 1-27). The histological grade was assessed according to the WHO 2004 criteria; 32 of the tumors $(58.2 \%)$ were low grade and $23(41.8 \%)$ were high grade. According to the UICC 2002 TNM classification system, 32 tumors were superficial $\left(\mathrm{Tis}, \mathrm{Ta}, \mathrm{T}_{1}\right)$ and 23 were invasive $\left(\mathrm{T}_{2}, \mathrm{~T}_{3}, \mathrm{~T}_{4}\right)$. Other clinical and pathological characteristics of the enrolled patients are shown in Table I. During the follow-up period, the recurrence or progression of BTCC was detected in 16 patients, 4 of whom succumbed to BTCC.

$R N A$ preparation and reverse transcription. Total RNA was isolated from 500-1,000 mg specimens of frozen bladder cancer tissue or normal bladder tissue using TRIzol reagent (Invitrogen, Carlsbad, CA, USA) according to the manufacturer's instructions. The quality of the RNA was confirmed to be high. Complementary DNA (cDNA) was synthesized from $2 \mu \mathrm{g}$ total RNA using random primers and Moloney murine leukemia virus (M-MLV) reverse transcriptase (Invitrogen).

Oligonucleotide primers for MTUS1 gene and amplification by reverse transcription-polymerase chain reaction $(R T-P C R)$. The specific oligonucleotide primers were synthesized according to published information on the MTUS1 gene (GenBank NM_001001924.2) as follows: sense, 5'-TGAGGCAAATAGCTGCTCCA-3'; antisense, 5'-TGAGGAGATACGGCTCGATCA-3'. The PCR product size was 106 bp. We conducted BLAST searches (GenBank) to confirm the specificity of the nucleotide sequences. To ensure the fidelity of mRNA extraction and reverse transcription, samples were subjected to PCR amplification with oligonucleotide primers specific for $\beta$-actin and normalized primers. $\beta$-actin primers were as follows: forward: 5'-CATGTACGTTGCTATCCAGGC-3'; reverse: 5'-CTCCTTAATGTCACGCACGAT-3'. The PCR product size of $\beta$-actin was $318 \mathrm{bp}$. The PCR conditions were as follows: denaturation at $95^{\circ} \mathrm{C}$ for $5 \mathrm{~min}$, followed by 35 cycles of $30 \mathrm{sec}$ at $94^{\circ} \mathrm{C}$, annealing at $57^{\circ} \mathrm{C}$ for $30 \mathrm{sec}$ at and at $72^{\circ} \mathrm{C}$ for $30 \mathrm{sec}$. The final extention was at $72^{\circ} \mathrm{C}$ for $5 \mathrm{~min}$. An $8-\mu 1$ aliquot of
Table I. Correlation between clinicopathological characteristics and expression of MTUS1 in bladder cancer.

\begin{tabular}{|c|c|c|c|}
\hline Variable & Case & $\begin{array}{l}\text { Relative expression of } \\
\text { MTUS } 1 \text { mRNA }( \pm \text { SD) }\end{array}$ & P-value \\
\hline Gender & & & 0.332 \\
\hline Male & 44 & $240 \pm 92$ & \\
\hline Female & 11 & $270 \pm 86$ & \\
\hline Age (years) & & & 0.357 \\
\hline$\leq 50$ & 13 & $226 \pm 82$ & \\
\hline$>50$ & 42 & $253 \pm 94$ & \\
\hline Grade & & & $<0.001^{\mathrm{a}}$ \\
\hline Low & 32 & $307 \pm 58$ & \\
\hline High & 23 & $161 \pm 51$ & \\
\hline T stage & & & $<0.001^{\mathrm{a}}$ \\
\hline Tis, Ta, $\mathrm{T}_{1}$ & 32 & $298 \pm 69$ & \\
\hline $\mathrm{T}_{2}, \mathrm{~T}_{3}, \mathrm{~T}_{4}$ & 23 & $174 \pm 66$ & \\
\hline Tumor number & & & $0.029^{\mathrm{a}}$ \\
\hline Single & 40 & $263 \pm 85$ & \\
\hline Multiple & 15 & $203 \pm 97$ & \\
\hline Tumor size $(\mathrm{cm})$ & & & $0.034^{\mathrm{a}}$ \\
\hline$\leq 3$ & 43 & $260 \pm 92$ & \\
\hline$>3$ & 12 & $197 \pm 73$ & \\
\hline
\end{tabular}

aStatistically significant. Grading according to 2004 WHO criteria; $\mathrm{T}$ staging according to 2002 UICC TNM classification. MTUS1, mitochondrial tumor suppressor 1 .

each amplified PCR product was electrophoresed on $1.5 \%$ (w/v) agarose gels containing $0.5 \mu \mathrm{g} / \mathrm{ml}$ ethidium bromide which were visualized under UV light. The band density was detected and evaluated using the Quantity One Quantization software (Bio-Rad, Munich, Germany).

Real-time RT-PCR and analysis of MTUS1 mRNA. The real-time quantitative RT-PCR amplification of MTUS1 and $\beta$-actin mRNA from the tissue samples was performed using the ABI 7500 Real-Time PCR system using the SYBR-GreenI kit (Takara Biotechnology, Dalian, China). Data were analyzed using the 7500 System SDS software (Applied Biosystems, Foster City, CA, USA). In brief, the PCR was carried out in a $50 \mu \mathrm{l}$ final volume containing a) $10 \mu \mathrm{l}$ 5X SYBR-GreenI PCR buffer; b) $1 \mu \mathrm{l}$ sense primer $(10 \mu \mathrm{M})$ and $1 \mu \mathrm{l}$ antisense primer $(10 \mu \mathrm{M})$; c) $1 \mu \mathrm{ldNTP}(10 \mathrm{mM})$; d) $1 \mu \mathrm{l}$ Taq enzyme (3 U/ $\mu \mathrm{l})$; e) $5 \mu \mathrm{l}$ cDNA; and f) $\mathrm{ddH}_{2} \mathrm{O}$ up to $50 \mu \mathrm{l}$. Following initial denaturation at $93^{\circ} \mathrm{C}$ for $3 \mathrm{~min}$, temperature cycling was initiated. Each cycle consisted of denaturation at $93^{\circ}$ for $30 \mathrm{sec}$, annealing at $55^{\circ} \mathrm{C}$ for $45 \mathrm{sec}$ and extension at $72^{\circ} \mathrm{C}$ for $45 \mathrm{sec}$. A total of 40 cycles was carried out. To distinguish specific from non-specific products and primer dimers, melting curve analyses were carried out. To evaluate specific mRNA expression in the samples, a standard curve was produced for each run. The concentration of each sample was calculated by relating its crossing point to a standard curve. The level of expression of MTUS1 mRNA is presented as relative copy numbers normalized against $\beta$-actin mRNA and shown as the 
A

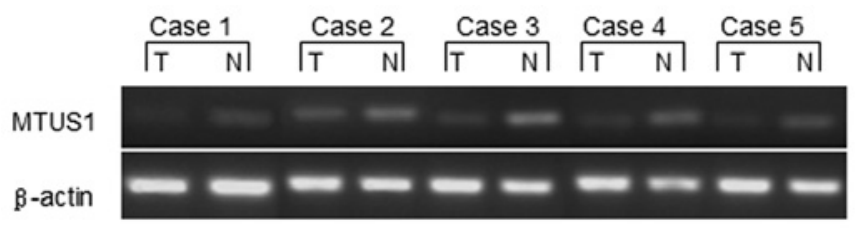

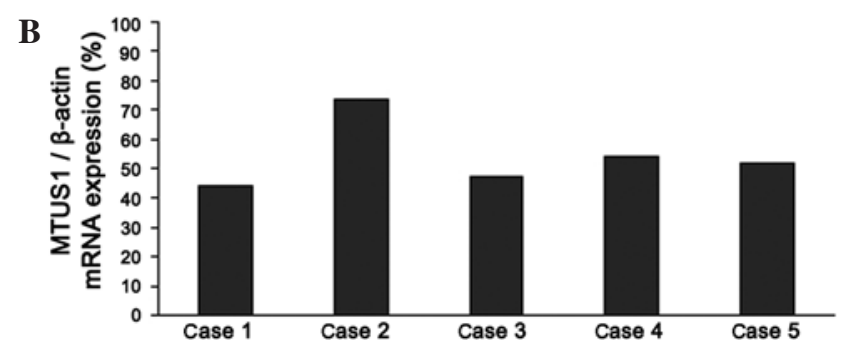

Figure 1. RT-PCR analysis comparing the MTUS1 mRNA expression in bladder tumor (T) and corresponding normal surrounding tissue (N) in 5 patients. (A) Agarose gel of RT-PCR. $\beta$-actin was used as an internal control. (B) Graphic analysis of the MTUS1 mRNA expression. Results were normalized to $\beta$-actin expression levels. MTUS1 expression in the normal tissue was defined as $100 \%$ in each patient. RT-PCR, reverse transcription-polymerase chain reaction; MTUS1, mitochondrial tumor suppressor 1.

mean \pm SD. Relative MTUS1 mRNA expression was calculated using the formula: (MTUS1/ $\beta$-actin) x 1,000.

Statistical analysis. The correlation between MTUS1 mRNA expression and clinicopathological factors was analyzed using the $\chi^{2}$ test and Student's t-test. For survival analysis, disease-free survival (DFS) was defined as the time interval from surgery to cancer recurrence or progression. DFS curves were generated using the Kaplan-Meier method and the comparison between the curves was carried out using the log-rank test. $\mathrm{P}<0.05$ was considered to indicate a statistically significant result. Statistical analysis was performed using SPSS 13.0 software for Windows (SPSS Inc., Chicago, IL, USA). Prognostic factors were evaluated by univariate and multivariate logistic regression analyses.

\section{Results}

MTUS1 mRNA expression in bladder cancer. We performed an RT-PCR analysis of MTUS1 in bladder tumors and paired normal samples obtained from 5 patients. A significant downregulation of MTUS1 mRNA expression was observed in the tumor tissues as compared with the corresponding normal bladder tissue (Fig. 1). The mean MTUS1 mRNA expression in the tumor tissues was $54.1 \%$ (range, 43.9-73.3\%) compared with the normal tissues (with expression in normal tissues defined as $100 \%$ ). We further confirmed the expression of MTUS1 mRNA in 55 bladder cancer samples and 10 paired normal samples by real-time RT-PCR analysis. The mean expression value of MTUS1 mRNA in the cancer samples was 246.1 \pm 91.3 , which was significantly lower than that for the normal samples $(405.3 \pm 124.4 ; \mathrm{P}<0.001)$.

Clinicopathological significance of MTUS1 mRNA expression in bladder cancer. The expression of MTUS1 mRNA in bladder cancer and its correlation with clinicopathological factors were examined (Table I). Four of these factors were positively correlated with the expression of MTUS1 mRNA. First, the level of MTUS1 mRNA expression was found to be marginally higher in the low-grade group $(307 \pm 58)$ compared to that in the high-grade group $(161 \pm 51 ; \mathrm{P}<0.001)$. Second, MTUS1 mRNA was expressed at higher levels in the superficial tumor group $(298 \pm 69)$ than in the invasive tumor group (174 $\pm 66 ; \mathrm{P}<0.001)$. Third, the MTUS1 mRNA expression level was higher in the group with a single mass $(263 \pm 85)$ than in the multiple mass group $(203 \pm 97 ; \mathrm{P}=0.029)$. Finally, there was also a significant correlation between levels of MTUS1

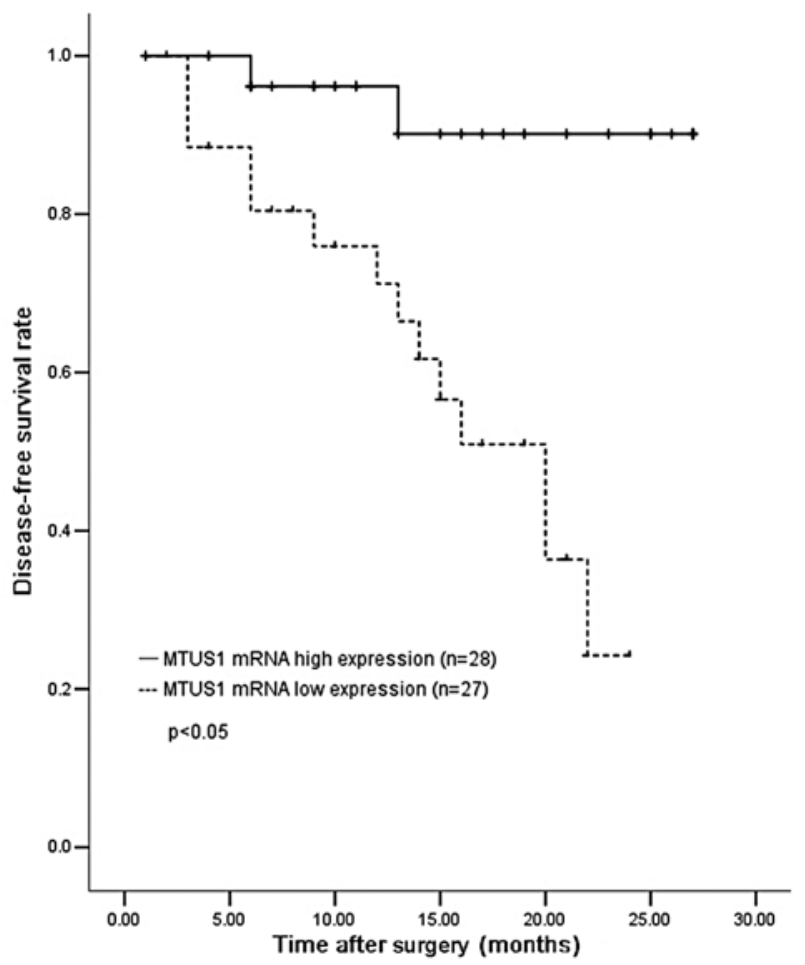

Figure 2. Disease-free survival curves of patients with bladder cancer according to the level of MTUS1 mRNA expression. There was a significant difference between the patients with high and those with low MTUS1 mRNA expression $(\mathrm{P}<0.05)$. MTUS1, mitochondrial tumor suppressor 1.

mRNA expression and tumor size; tumors $<3 \mathrm{~cm}$ showed a

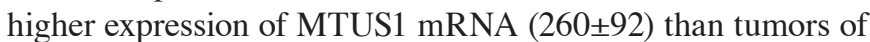
$>3 \mathrm{~cm}(197 \pm 73 ; \mathrm{P}=0.034)$. However, no differences were found between MTUS1 mRNA expression and age $(\mathrm{P}=0.357)$ or gender $(\mathrm{P}=0.332)$. In addition, MTUS1 mRNA expression was lower in the 16 patients who experienced recurrence or progression following surgery compared with those who did not; the mRNA expression mean levels were $164 \pm 65$ and $280 \pm 78$, respectively $(\mathrm{P}<0.001)$.

Prognostic value of MTUS1 mRNA expression for DFS. We evaluated whether MTUS1 mRNA expression was able to predict tumor recurrence or progression in bladder cancer. The cases were divided into high $(n=28)$ and low $(n=27)$ expression groups according to the average MTUS1 mRNA expression status in the tumor. The cut-off value was the most significant 
Table II. Univariate and multivariate logistic regression analysis for disease-free survival.

\begin{tabular}{lcrc}
\hline & Univariate analysis & & Multivariate analysis \\
\cline { 3 - 4 } Variable & P-value & P-value & RR \\
\hline Gender & 0.824 & & \\
Age (years) & 0.230 & & 0.460 \\
Grade & $0.001^{\mathrm{a}}$ & 0.561 & 0.561 \\
T stage & $0.009^{\mathrm{a}}$ & 0.556 & $0.034-6.309$ \\
Tumor number & $0.037^{\mathrm{a}}$ & 0.630 & 0.573 \\
Tumor size (cm) & 0.995 & & $0.249-9.958$ \\
MTUS1 expression & $<0.001^{\mathrm{a}}$ & $0.025^{\mathrm{a}}$ & 0.974
\end{tabular}

${ }^{\text {aS }}$ tatistically significant. RR, relative risk; CI, confidence interval. Grading according to 2004 WHO criteria; T staging according to 2002 UICC TNM classification. MTUS1, mitochondrial tumor suppressor 1.

Table III. Multivariate logistic regression analysis of factors associated with expression of MTUS1 mRNA.

\begin{tabular}{llll}
\hline Variable & P-value & RR & \multicolumn{1}{c}{$95 \%$ CI } \\
\hline Gender & 0.957 & 0.925 & $0.055-15.470$ \\
Age & 0.159 & 9.238 & $0.419-203.892$ \\
Grade & $0.001^{\mathrm{a}}$ & 0.005 & $0.000-0.114$ \\
T stage & $0.046^{\mathrm{a}}$ & 0.060 & $0.004-0.953$ \\
Tumor number & 0.416 & 0.300 & $0.017-5.460$ \\
Tumor size & 0.522 & 2.870 & $0.114-72.351$
\end{tabular}

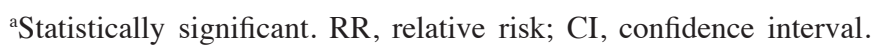
Grading according to $2004 \mathrm{WHO}$ criteria; T staging according to 2002 UICC TNM classification. MTUS1, mitochondrial tumor suppressor 1 .

value for prognostic prediction by the log-rank test. Patients with a high MTUS1 expression (mean, 321 \pm 50 ) had a significantly longer DFS than those with a low expression (mean, $169 \pm 51 ; \mathrm{P}<0.05$ ) (Fig. 2).

Univariate and multivariate prognostic analyses of DFS in bladder cancer. The results of the univariate and multivariate prognostic analyses of postoperative DFS are shown in Table II. Univariate analysis revealed that the following factors were significantly correlated with DFS: grade $(\mathrm{P}=0.001)$, $T$ stage $(\mathrm{P}=0.009)$, tumor number $(\mathrm{P}=0.037)$ and MTUS1 mRNA expression $(\mathrm{P}<0.001)$. Multivariate regression analysis revealed that MTUS1 mRNA expression was an independent prognostic predictor for DFS [relative risk (RR), 0.974; $95 \%$ confidence interval (CI), 0.952-0.997; $\mathrm{P}=0.025$ ].

To clarify the most significant factors correlated with MTUS1 mRNA expression in bladder cancer, we performed multivariate analyses. Results of the multivariate logistic regression analysis demonstrated that grade and stage of the disease were significantly associated with MTUS1 mRNA expression in bladder cancer $(\mathrm{P}=0.001$ and $\mathrm{P}=0.046$, respectively) (Table III).

\section{Discussion}

The MTUS1 gene contains 17 coding exons that are distributed over $112 \mathrm{~kb}$ of genomic DNA. The use of alternative exons produces three major transcripts, termed ATIP1, ATIP3 and ATIP4, which show different tissue distributions (14). ATIP1 is ubiquitous and highly expressed in the brain, ATIP3 is expressed in most tissues, including the prostate, bladder, breast, ovary and colon, and ATIP4 is a brain-specific transcript that is highly abundant in the cerebellum and fetal brain.

In the several splice variants of MTUS1, ATIP3 is the major transcript which is localized to the centrosome, mitotic spindle and intercellular bridge. ATIP3 regulates the essential steps of mitosis by interfering with the microtubule cytoskeleton and its overexpression delays the progression of mitosis by prolonging the duration of the metaphase, potentially due to the modulation of spindle checkpoint signaling. ATIP3 knock-down by siRNA has been reported to lead to a significant increase in breast cancer cell proliferation, indicating an antiproliferative effect of ATIP3 (15).

The MTUS1 gene encodes a protein with a C-terminal domain, which interacts with the AT2 receptor, and a large coiled-coil region that facilitates dimerization. The AT1 and AT2 receptors belong to the superfamily of $G$ protein-coupled receptors (GPCR) and are the two major angiotensin II (ANG II) receptor subtypes. ANG II is not only a potent vasoactive effector peptide of the renin-angiotensin system, but also a significant regulator of cell proliferation and hypertrophy. AT1 and AT2 receptors exhibit opposite biological and physiological effects. MTUS1 is an early mediator of AT2 receptor activation. Together with AT2, MTUS1 antagonizes AT1 receptor function, inducing antiproliferative and pro-apoptotic effects in vitro and in vivo (16).

In the present study, we used semi-quantitative and quantitative RT-PCR to investigate the level of MTUS1 (ATIP3) mRNA expression in clinical cases of BTCC. We found that the expression of MTUS1 was low in the cancer tissues compared with the normal bladder tissues (Fig. 1). Previous studies have shown that MTUS1 expression levels are downregulated in cancers of the colon, ovary, pancreas, head and neck and breast cancer $(5,6,17-19)$. For example, Zuern et al reported 
that MTUS1 expression is significantly downregulated in colon cancer tissues, compared with the corresponding normal tissues, at the protein and mRNA levels (20). The authors also found that knockdown of MTUS1 in HUVEC cells by siRNA transfection resulted in increased cell proliferation. Pils et al reported similar results in ovarian carcinoma (6), as MTUS1 had a significantly lower expression in primary ovarian carcinoma compared with normal ovarian tissues and cysts. In addition, other studies have revealed the downregulation of MTUS1 in colon cancer and MTUS1 copy number deletion variants in familial breast cancer $(17,19)$. These data support our results. However, no studies have focused on bladder cancer and our analysis explored the clinical significance of MTUS1 expression in this disease.

We compared various clinicopathological factors with the MTUS1 expression status in bladder cancer. Our data demonstrate that the cases with high levels of MTUS1 mRNA expression tended to show low grade, low stage, small tumor size and a single tumor mass compared with those with low MTUS1 expression tumors (Table I). The correlation of expression with clinicopathological features provides clinical evidence that MTUS1 is a bladder tumor suppressor gene.

Univariate analysis in our study demonstrated that the following factors were prognostic for recurrence or progression: grade, T stage, tumor number and MTUS1 mRNA expression (Table II). Multivariate analysis further identified MTUS1 mRNA expression as a stronger independent prognostic factor of DFS than grade, $\mathrm{T}$ stage and tumor number in the logistic regression model. Thus, we suggest that MTUS1 is significant in the pathology of bladder cancer. The low level of expression of MTUS1 in bladder cancer was shown to be significantly associated with a poor DFS prognosis (Fig. 2). Therefore, the downregulation of MTUS1 expression may be an early event in malignant disease. In addition, grade and stage were significantly associated with MTUS1 mRNA expression in bladder cancer using multivariate analysis (Table III). Based on these results, the detection of MTUS1 in tumor tissue following surgery may be used as a prognostic marker for determining the risk of future recurrence or progression in patients with bladder cancer.

The exact mechanism by which MTUS1 regulates cell proliferation is currently unclear, but MTUS1 is believed to be an early component of the growth inhibitory signaling cascade. Seibold et al studied MTUS1 mRNA expression in pancreatic tumors and tumor cell lines (5). The authors reported a negative correlation of MTUS1 mRNA expression with cell proliferation and differentiation, showing low expression in undifferentiated proliferating cells and high expression in differentiated and slowly proliferating cells. This study also demonstrated an inhibitory effect of MTUS1 on cell proliferation by transfecting a recombinant plasmid containing the MTUS1 gene into a pancreatic tumor cell line which expressed no native MTUS1 mRNA. Moreover, the antigrowth effects of MTUS1 in cooperation with AT2 may be associated with the activation of tyrosine phosphatases and the inhibition of receptor tyrosine kinases (RTK), including bFGF, EGF and insulin, which ultimately lead to the inhibition of extracellular regulated kinase (ERK2) activation (21). The members of the RTK signaling pathway and ERK2 are known to be associated with carcinogenesis $(22,23)$.
To the best of our knowledge, this is the first study concerning correlations between MTUS1 expression and clinicopathological factors in bladder cancer. However, the clinical significance of MTUS1 mRNA expression should be further studied with regard to MTUS1 protein levels in bladder cancer. Further function studies are needed to elucidate the mechanism of tumor suppression of MTUS1 and to confirm its tumor suppression function in other bladder tumor types and models. Of note, the total sample size included in this study, was small, the follow-up duration was relatively short and the correlation between MTUS1 and overall survival was absent. Another study with a larger study population and longer follow-up on this issue is ongoing.

In conclusion, our study provides clinical evidence which supports the hypothesis that MTUS1 is a bladder cancer suppressor gene that may be significant in cancer development and progression. The status of MTUS1 mRNA expression could be a novel prognostic marker for predicting bladder tumor DFS.

\section{References}

1. Jemal A, Bray F, Center MM, Ferlay J, Ward E and Forman D: Global cancer statistics. CA Cancer J Clin 61: 69-90, 2011.

2. Ploeg M, Aben KK and Kiemeney LA: The present and future burden of urinary bladder cancer in the world. World J Urol 27: 289-293, 2009.

3. Lei T, Mao WM, Yang HJ, et al: Study on cancer incidence through the Cancer Registry Program in 11 cities and counties, China.Zhonghua Liu Xing Bing Xue Za Zhi 30: 1165-1170, 2009 (In Chinese).

4. Jacobs BL, Lee CT and Montie JE: Bladder cancer in 2010: how far have we come? CA Cancer J Clin 60: 244-272, 2010.

5. Seibold S, Rudroff C, Weber M, Galle J, Wanner C and Marx M: Identification of a new tumor suppressor gene located at chromosome 8p21.3-22. FASEB J 17: 1180-1182, 2003.

6. Pils D, Horak P, Gleiss A, et al: Five genes from chromosomal band 8 p22 are significantly down-regulated in ovarian carcinoma: $\mathrm{N} 33$ and EFA6R have a potential impact on overall survival. Cancer 104: 2417-2429, 2005.

7. Dexter TJ, Sims D, Mitsopoulos C, Mackay A, Grigoriadis A, Ahmad AS and Zvelebil M: Genomic distance entrained clustering and regression modelling highlights interacting genomic regions contributing to proliferation in breast cancer. BMC Syst Biol 4: 127, 2010.

8. Sayagués JM, Abad Mdel M, Melchor HB, et al: Intratumoural cytogenetic heterogeneity of sporadic colorectal carcinomas suggests several pathways to liver metastasis. J Pathol 221: 308-319, 2010.

9. Fong Y, Lin YS, Liou CP, Li CF and Tzeng CC: Chromosomal imbalances in lung adenocarcinomas with or without mutations in the epidermal growth factor receptor gene. Respirology 15: 700-705, 2010.

10. Califano D, Pignata S, Pisano C, et al: FEZ1/LZTS1 protein expression in ovarian cancer. J Cell Physiol 222: 382-386, 2010.

11. Cheng L, MacLennan GT, Zhang S, et al: Evidence for polyclonal origin of multifocal clear cell renal cell carcinoma. Clin Cancer Res 14: 8087-8093, 2008.

12. Kim JW, Cheng Y, Liu W, et al: Genetic and epigenetic inactivation of LPL gene in human prostate cancer. Int J Cancer 124: 734-738, 2009.

13. Knowles MA, Aveyard JS, Taylor CF, Harnden P and Bass S: Mutation analysis of the $8 \mathrm{p}$ candidate tumour suppressor genes DBC2 (RHOBTB2) and LZTS1 in bladder cancer. Cancer Lett 225: 121-130, 2005 .

14. Di Benedetto M, Bièche I, Deshayes F, et al: Structural organization and expression of human MTUS1, a candidate 8p22 tumor suppressor gene encoding a family of angiotensin II AT2 receptor-interacting proteins, ATIP. Gene 380: 127-136, 2006.

15. Rodrigues-Ferreira S, Di Tommaso A, Dimitrov A, et al: 8p22 MTUS1 gene product ATIP3 is a novel anti-mitotic protein underexpressed in invasive breast carcinoma of poor prognosis. PLoS One 4: e7239, 2009. 
16. Stoll $\mathrm{M}$ and Unger T: Angiotensin and its AT2 receptor: new insights into an old system. Regul Pept 99: 175-182, 2001.

17. Lee S, Bang S, Song K and Lee I: Differential expression in normal-adenomacarcinoma sequence suggests complex molecular carcinogenesis in colon. Oncol Rep 16: 747-754, 2006

18. Ye H, Pungpravat N, Huang BL, et al: Genomic assessments of the frequent loss of heterozygosity region on 8p21.3-p22 in head and neck squamous cell carcinoma. Cancer Genet Cytogenet 176: 100-106, 2007.

19. Frank B, Bermejo JL, Hemminki K, et al: Copy number variant in the candidate tumor suppressor gene MTUS1 and familial breast cancer risk. Carcinogenesis 28: 1442-1445, 2007.
20. Zuern C, Heimrich J, Kaufmann R, et al: Down-regulation of MTUS1 in human colon tumors. Oncol Rep 23: 183-189, 2010.

21. Nouet S, Amzallag N, Li JM, et al: Trans-inactivation of receptor tyrosine kinases by novel angiotensin II AT2 receptor-interacting protein, ATIP. J Biol Chem 279: 28989-28997, 2004.

22. Vogelstein B and Kinzler KW: Cancer genes and the pathways they control. Nat Med 10: 789-799, 2004.

23. Meloche S and Pouysségur J: The ERK1/2 mitogen-activated protein kinase pathway as a master regulator of the G1- to S-phase transition. Oncogene 26: 3227-3239, 2007. 